\title{
Aligning Goal Analysis and Business Process Modelling: A Case Study in Health Care
}

\author{
Evellin C.S. Cardoso, Renata S.S. Guizzardi and \\ João Paulo A. Almeida \\ Ontology and Conceptual Modeling Research Group (NEMO) \\ Computer Science Department, Federal University of Espírito Santo (UFES), \\ Brazil
}

ecardoso@inf.ufes.br

rguizzardi@inf.ufes.br

jpalmeida@ieee.org

\begin{abstract}
This paper discusses the integration of goal analysis and business process modelling by using, respectively, Tropos and ARIS for each of these activities. The main benefit of the approach relates to traceability between goal models and business process models. The approach is exemplified with a case study in a health care organization. We have observed that establishing the relations between goal and business process models is far from straightforward. This can be accounted by the fact that goals may be formulated in various levels of abstraction and precision and further, that goals may refer to various aspects of an organization and its processes. Our approach for alignment is based on harmonizing goal models such that they can be subsequently aligned with business process models. We propose a goal taxonomy to harmonize the goal domain and report on the implications of this classification in establishing the relationships with business processes.
\end{abstract}

Keywords: Goal Analysis; Business Process Modelling; Health Care Organizations; Tropos; ARIS.

\begin{abstract}
Biographical notes: The authors of this paper are all members of the Ontology and Conceptual Modeling Research Group (NEMO), which is part of the Computer Science Department of the Federal University of Espírito Santo, Brazil. Evellin C.S. Cardoso holds an M.Sc. in Computer Science from the Federal University of Espírito Santo. Her main research interests include Conceptual Modelling, Enterprise Architecture and Agentoriented Software Engineering. Dr. Renata S.S. Guizzardi is lecturer at the Federal University of Espírito Santo. Her research interests include Knowledge Management, Agent-oriented Software Engineering and Organizational Modelling. Dr. João Paulo A. Almeida is lecturer at the Federal University of Espírito Santo. His key research areas include: Model-driven Design, Service-oriented Design, Enterprise Architecture, Conceptual Modelling and Business Process Modelling.
\end{abstract}

\section{INTRODUCTION}

Business process modelling basically comprises an activity whose main goal is to provide a formalization of business processes in an organization or a set of cooperating organizations. By modelling an organization's business processes, it is possible to capture how the organization aims at achieving its goals and strategies.

Since business processes and goals are intrinsically interdependent, establishing an alignment between the process and the goal domains arises as a natural approach. While the goal domain copes with selecting and prioritizing organizational strategies, the business process domain addresses the execution of organizational activities, possibly revealing the computational support provided by information systems to these activities.

The main benefit of the alignment between the goal and business process domains relates to traceability between goals and business process models [9] [1512], i.e., our 
ability to account for how strategies are operationalized into business processes, and to account for how business processes impact the achievement of goals. Traceability between goal models and business process models is particularly important for business process reengineering to support the selection between alternative process changes, since the strategies that must be achieved in TO-BE business processes may be documented in goal models [22].

Further, if business process models are to be used as a starting point for the definition of process-oriented information systems [4], then the alignment between goals and business process directly affects the alignment of information systems and organizational goals and strategies. In other words, goal models play an essential role in increasing the quality of business process models by incorporating the notion of intentionality in these models. Therefore, the alignment between business process models and goal models promotes the development of processoriented information systems which are fully aligned with organizational goals.

In this work, we adopt the Tropos methodology [2][3] for the elaboration of goal models and the ARIS framework [21] for business process modelling. The Tropos methodology facilitates goal analysis through a language which is expressive enough to capture complex relationships between goals and which is able to capture dependency relationships among organizational agents [2]. Further, Tropos provides sophisticated tools for contribution analysis and formal reasoning for assessing goal satisfaction [7]. The ARIS framework, in its turn, provides marginal support for goals in its Objectives Diagram but enables detailed representation of business processes and organizational structures.

We conducted a case study on the alignment of goals and business processes in the Rheumatology Department of a hospital in Brazil. In this context, we developed both goal models and business process models through interviews and active observations, investigating the relations between them. As a result of this study, we observed that establishing the relations between goals and business process models is far from straightforward. This can be accounted by the fact that goals may be formulated in various levels of abstraction [1918] and precision [11] and, further, that goals may refer to various aspects of an organization and its processes. Our approach for alignment is based on harmonizing goal models such that they can be subsequently aligned with business process models. We propose a goal taxonomy to harmonize the goal domain and report on the implications of this classification in establishing the relationships with business processes.

This paper is structured as follows: section 2 describes the methodologies used in the alignment and discusses the potential benefits of alignment; section 3 presents the case study which reports our experience in the Rheumatology Department of a hospital in Brazil; section 4 discusses related work and finally, section 5 presents our conclusions.

\section{ALIGNING GOAL ANALYSIS AND BUSINESS PROCESS MODELLING}

This section describes the methodologies used in this paper: section 2.1 presents the Tropos methodology for goal analysis; section 2.2 focuses on the ARIS methodology for business process modelling. Further, section 2.3 discusses the main benefits which stem from the alignment between both methodologies.

\subsection{Goal Analysis with Tropos methodology}

The $i^{*}$ framework, initially proposed in Yu's $\mathrm{PhD}$ Thesis [22], consists in an agent-oriented conceptual framework whose focus is on intentional characteristics of organizational actors. The Tropos methodology has been conceived with basis on the $i^{*}$ framework and adopts the same concepts in early requirements stages for software development [2]. In this paper, we use the Tropos methodology and terminology for goal modelling.

The goal modelling technique starts with a focus on the actors of the domain. In this stage, we mainly focus on organizational aspects which characterize these actors as social agents. Generally, goal analysis aims at answering some questions such as: "What are the main strategic goals of some organization?", "Who adopts these objectives?", "Which alternative solutions are regarded in order to achieve these strategies?" and "Which aspects must be addressed in the decision making process?"

With respect to goals, Tropos relies on two primitives for goal modelling: hardgoals and softgoals. The language has a general concept goal which, in its turn is refined into these two concepts.

A goal, according to [22], is defined as a condition or state of affairs in the world that the actor would like to achieve. Hardgoals are defined as goals whose satisfaction can be objectively defined [2]. Softgoals are the opposite of hardgoals, since they are "subject to interpretation" [22], "imprecise, subjective, context-specific, and ideal" [11]. This different nature of achievement is denoted in the terms used for stating fulfilment: it is said that hardgoals are satisfied while softgoals are satisficed [18]. Besides goals, actors also have plans and resources. The relationship between plans and goals rests on the fact that goals represent "a set of states of affairs (i.e. a set of world states)" [8], while plans "represent, at an abstract level, a way of doing something. The execution of plan can be a means for satisfying a goal or for satisficing a softgoal." [2].

For modelling relationships between the Tropos's primitives, three types of relationships among them are provided: means-end link, contribution link, and AND/OR decomposition. In particular, means-end analysis aims at capturing goals, plans, resources and softgoals that provide means for achieving a goal. Contribution analysis identifies goals that can contribute positively or negatively in the attainment of the goal to be analyzed. An AND decomposition supports a goal to be decomposed in a series of sub-goals; while an OR decomposition allows modelling of alternative ways of achieving a goal. Besides, temporal 
logic specifications can be used to specify constraints on the models.

When all primitives mentioned above (hardgoals, softgoals, plans and resources) are assigned to a specific actor, they are represented in a goal diagram which comprises, basically, in an internal perspective of an organizational actor. Although this goal diagram can be used to capture actors' concerns and motivations, commonly, these actors cannot satisfy their goals in isolation and therefore, actors are required to establish dependencies which are represented in actor diagrams. Dependencies allow actors to attain goals which would not be possible on their own, or not as easily, or not as well [2].

One of the main advantages of applying the Tropos methodology in organizational environments is the opportunity of noticing particularities of the environment, such as: (a) the verification of inconsistencies between models elaborated from the point of view of different stakeholders; (b) the detection of tasks performed by multiple organizational actors repeatedly, which suggests that the efficiency of the business process can be improved if the issue is addressed (c) the understanding that little attention is devoted either to collaborative activities or knowledge-intensive activities; (d) the detection of problems related with the lack or the inadequacy of policies and/or information systems (e.g. lack of thrust among actors, gap between business process and information system, etc.) and (e) the establishment of dependency relationships among actors in the achievement of their goals (this helps the detection of non-reciprocal relationships among actors, revealing actors' vulnerabilities).

\subsection{ARIS Methodology for business process modelling}

ARIS (ARchitecture for integrated Information Systems) has been developed in Saarbrucken (Germany), in 1992, with the main aim at providing an architectural framework for enterprise description. The framework is composed of three abstraction levels (Requirements Definition, Design Specification and Implementation Description) and four viewpoints (Organizational, Control, Function and Data) which support the description of different enterprise domains and their relationships [21].

In this work, we adopt the ARIS framework for business process modelling (in ARIS framework, business process modelling belongs to the Requirements Definition level of abstraction and to the Control viewpoint). The business process modelling technique starts with capturing the Value-Added Chain (VAC) which represents all macroprocesses which are executed in order to achieve the organizational strategies.

Since macro-processes represent a bundle of business processes, the refinement of macro-processes produces a chain of processes which represent organizational procedures. A business process is a systematic sequence of related actions which produce measurable results by consuming inputs of varied nature. A business process may be triggered by events which are internal or external to the organization. In ARIS framework, business processes are modelled in diagrams denominated EPCs (Event-driven Process Chains) [21].

Figure 1 represents the refinement of the business process "Diagnosis the patient health state" shown in an EPC. In these diagrams, there are two notational elements to represent actors who perform some business process: ellipses when actors are organizational units or rectangles when they represent roles performed by human agents in the execution of business process. The activities executed by each actor are placed in his/her respective swimlane and are symbolized by green rectangles while the events are symbolized by pink hexagons. There are also logical operators which determine the flow of execution. For instance, in Figure 1, after the execution of the activity "Verify the previous achievement of laboratorial exams", the flow control can follow only one of the branches associated with either the event "Laboratorial exams not previously achieved" or the event "Laboratorial exams previously achieved" (exclusive-OR logical operator).

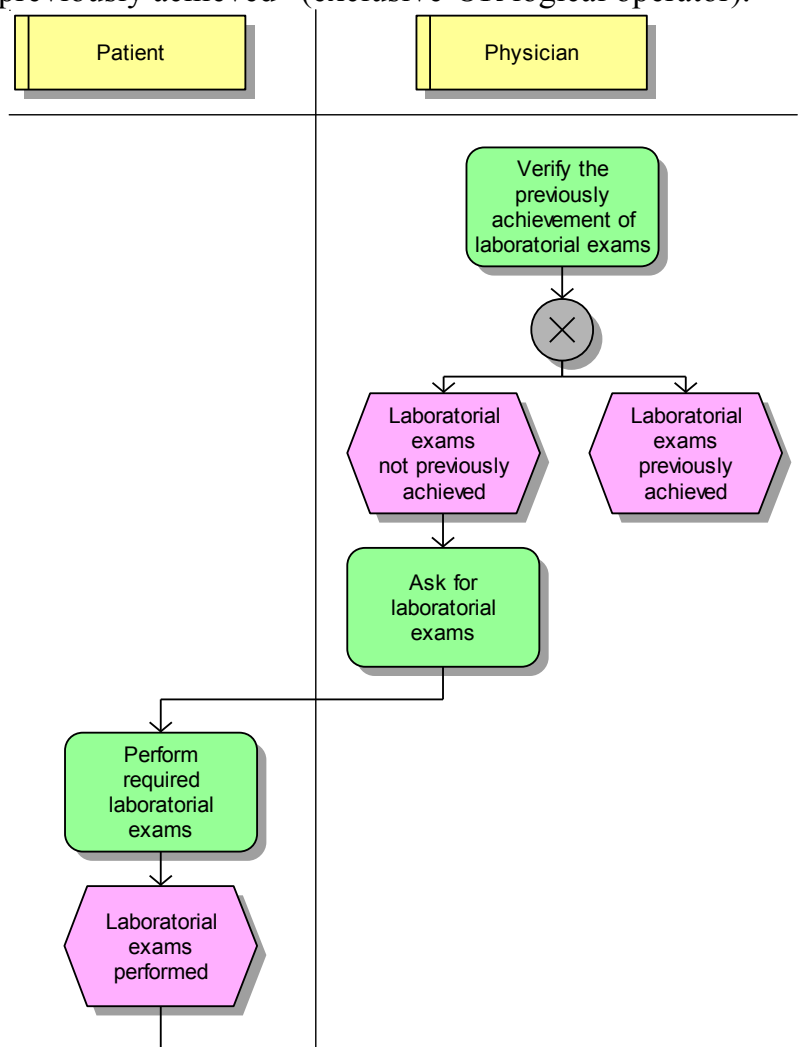

Figure 1. A fragment of the "Diagnose patients' health state" process model

When activities in a business processes can be described as a single action without further decomposition, Function Allocation Diagrams (FADs) are created for each activity. In an FAD, one can assign resources to the execution of actions, revealing the organizational units where the activities occur, their executors, the systems which support them, the incoming and outgoing documents and information, business rules, business requirements and risks associated with them. Despite the usefulness of these diagrams in subsequent stages of the methodology proposed in this work, the analysis of this kind of diagram is out of scope of this article. 


\section{CASE STUDY}

\subsection{Motivation}

We conducted a case study to investigate the relationship between the business process domain and the goal domain in a real-life scenario. The case study was conducted in the Rheumatology Department of the Cassiano de Moraes University Hospital (HUCAM), which is part of the Federal University of Espírito Santo in Vitória, Brazil.

In the hospital, the department has the following functions: (i) providing educational training to form specialists in rheumatology, (ii) providing outpatient medical care and (iii) developing research to investigate the incidence of rheumatologic conditions in population. The department include six specialists in rheumatology, two nurses and two physiotherapists, among other professionals for hosting patients. The department performs 15 business processes, such as outpatient care, drugs infusion, among others, and performs an average rate of 5700 outpatient medical care by year.

In the next sections, the outcomes of this case study are partially reported.

\subsection{Rheumatology Department's goals}

As can be seen in Figure 2, the Rheumatology Department has three main actors: the Patient, the Resident in Rheumatology and the Rheumatologist Physician (which are referred to as Patient, Resident and Physician, respectively in the remainder). The Patient has two main goals: Get healed and Feel well. These goals exemplify the distinction between hardgoal and softgoal. While one can objectively infer whether the patient is healed (from a physiological perspective through exams analysis), it is not possible to accurately determine what characterizes the patient's well-being. The Resident and the Physician aim at healing the patient (Heal the patient goal) and when this is not feasible, at least minimizing patient's physical suffering (Minimize patient's physical suffering and symptoms goal). This softgoal is important in this particular case, since in Rheumatology, in most of cases, the conditions are chronic and incurable, which implies in adopting a treatment which focuses on minimizing the condition effects.

In the scenario analyzed, in most of cases (but not in all cases), the medical consultation is not directly executed by the Physician, but by the Resident. This is captured by the dependency between the Patient and the Resident/Physician to obtain a medical consultation. In counterpart, the Resident/Physician depends on the Patient to acquire technical skills (Acquire technical skills goal dependency). Although the resident and physician have the same goal of acquiring technical knowledge in treating the patient, this goal has a different meaning for each actor. The Physician is interested in acquiring technical knowledge for becoming more experienced (Become more experienced goal) to treat the future cases in the course of his/her professional exercise. The Resident besides considering this issue is also concerned about being approved in an exam after concluding the residence to be acknowledged as a Rheumatology Specialist (the Acquire technical skills goal is also refined in Resident's perspective, not shown in this figure). In this sense, the Resident's dependency is more critic than the Physician's dependency on the Patient.

To satisfy legal requirements, after the Resident has analyzed the patient's case, he/she contacts the Physician to approve the diagnosis and proposed treatment. The Approve the treatment proposed by the resident goal dependency from the Resident towards the Physician captures this practice. The Physician in turn, depends on the Resident to Treat more patients. This is a matter of scaling up the service provided to patients. While there is just one physician in each shift, there are usually four residents.

This diagram enables us to consider the balance in the relationships between pairs of actors. This is relevant because mutual dependency leads to greater motivation for cooperation when compared to the cases that dependencies occur just in one direction. In the first case, both actors act towards establishing commitments which lead to adopting the goals of each other. The documentation of some unbalance (which does not occur here) in an AS-IS model open up the possibility of improvements to the generation of a TO-BE model. In other words, the analysis allows one to identify the cases in which it is necessary to modify organizational practices so that mutual dependencies are established.

After an abstract vision of the scenario under consideration, we focus on the particular goals of actors, deepening the analysis about their intentions, choices, resources and strategies to reach a specific goal. This analysis can be conducted using an actor diagram which shows the goals of a physician who conducts the business process of diagnosis, as exemplified in Figure 3. 


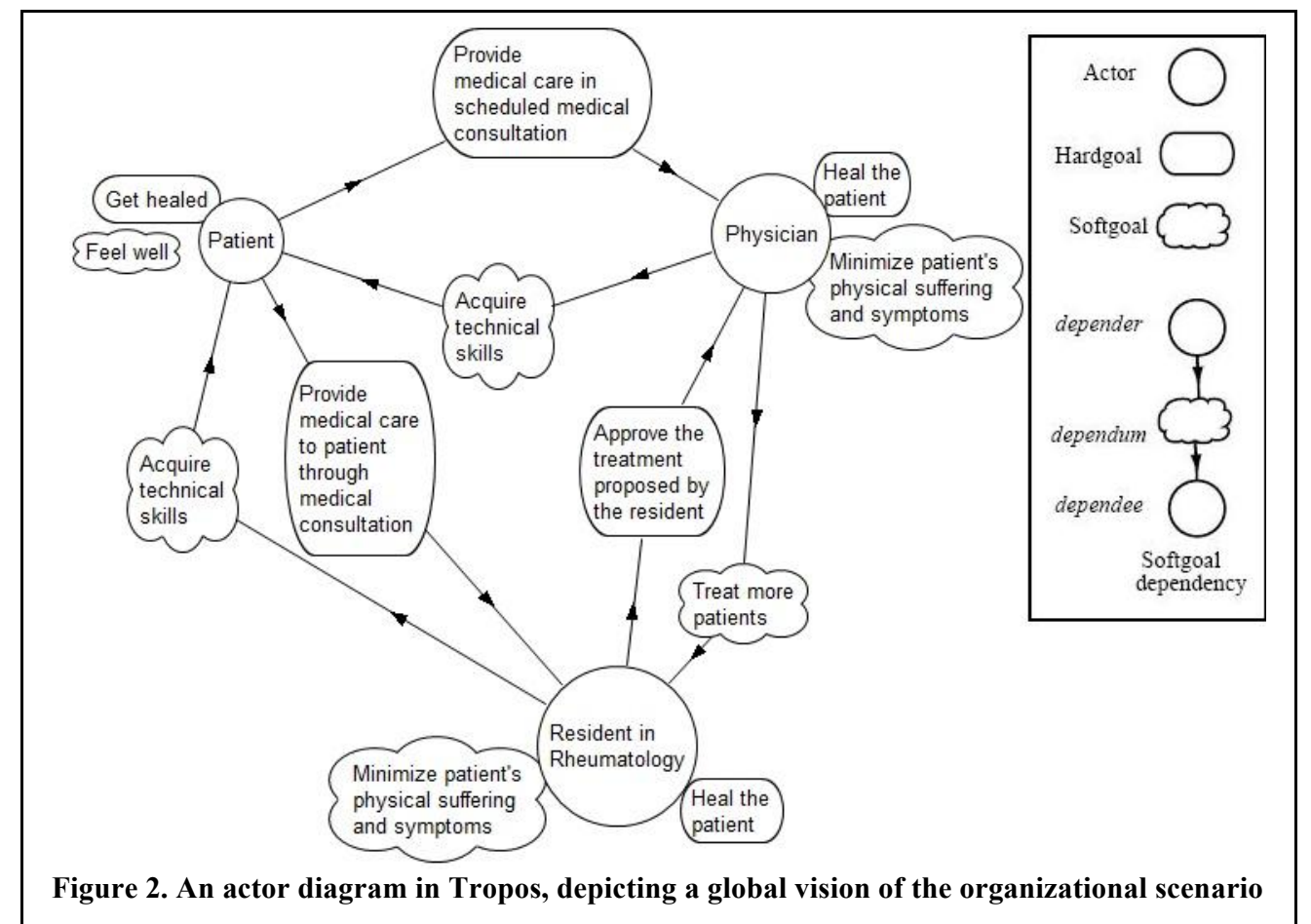

The first step in the analysis is to internalize the goals of the actor. We notice that the main goals of the Physician (Heal the patient and Minimize patient's physical suffering and symptoms) are internal to his/her perspective. Besides the actor's individual goals, it is necessary to internalize those goals which had been delegated from the dependencies established with other actors as shown in the actor diagram. Thus, the Provide medical care to patient through medical consultation goal delegated by the Patient is now represented inside the Physician's perspective. In the same way, the Physician assumes the Approve the treatment proposed by the resident goal, delegated by the Resident.

From this point on, we can identify how goals are interrelated. For example, one of the alternatives when a physician needs to provide medical care to a patient (Provide medical care to patient goal) is scheduling a medical consultation (Provide medical care in a scheduled medical consultation goal). This is captured by an OR-decomposition, in which the other alternative consists on the goal Provide medical care via ER. Providing medical care to patient contributes positively to minimize patient's physical suffering and symptoms
(Minimize patient's physical suffering and symptoms goal).

In the remainder of the section, we describe the goals with respect to a specific medical consultation. During consultation, the physician diagnoses the patient's health state (Diagnose health state goal) and prescribes the treatment (Prescribe patient's treatment goal which uses, in turn, a Drugs prescription).

The main goal of the physician is to Diagnose patient's health state. During the process of diagnosis, the physician can find either rheumatologic or non-rheumatologic conditions (Diagnose rheumatologic conditions goal and Diagnose non-rheumatologic conditions goal). Rheumatologic conditions can be, in turn, classified as mild conditions (Diagnose mild rheumatologic conditions goal) or serious conditions (Diagnose serious rheumatologic conditions goal). If a mild rheumatologic condition has been identified, the patient receives treatment for a short period of time and is soon released. This justifies the existence of the Release patients with mild rheumatologic conditions after no more than 3 consultations goal. 


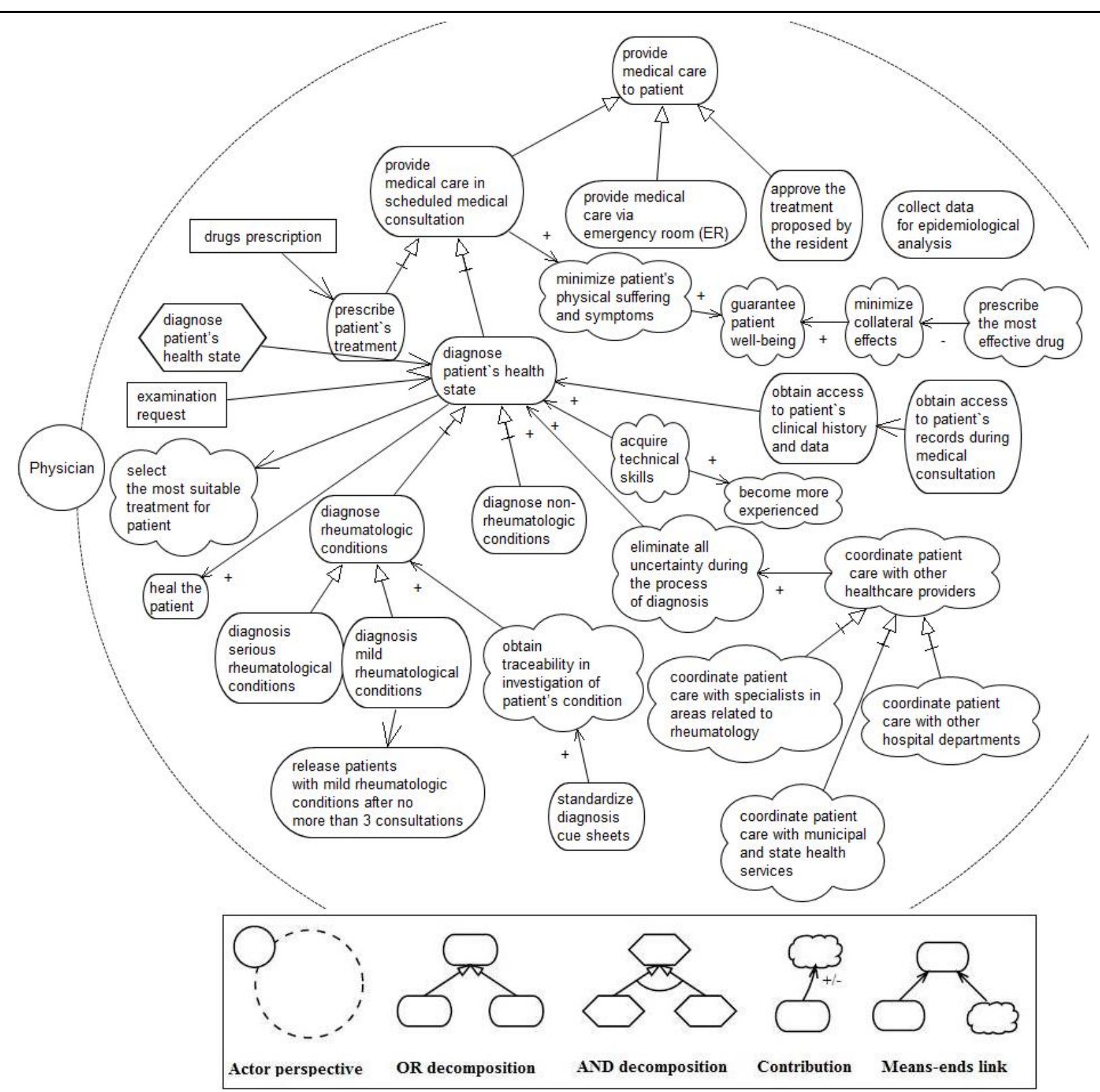

Figure 3. Goal diagram in Tropos which focuses on the Physician's perspective

After diagnosing the patient's heath state, the physician is able to select the most suitable treatment for the condition (Select the most suitable treatment for patient softgoal). For this reason, Diagnose patient's health state is a mean for Select the most suitable treatment for patient. With respect to the diagnosis, the physician is also interested in obtaining information about all the conditions which had already been previously investigated (Obtain traceability in investigation of patient's condition softgoal). One of many means towards achieving traceability in the investigation of diseases is to Standardize diagnosis cue sheets.

The physician must have accurate knowledge for being able to discover the presence/absence of conditions (Acquire technical skills softgoal), i.e., to become more experienced so as to discover conditions in future cases (Become more experienced goal). He/she must also access the patient's data for achieving the current medical consultation (Obtain access to patient's records during medical consultation goal). In the case which the physician is not able to access the patient's records, he/she has the legal right to deny providing assistance to patient. After analyzing this data, the physician is able to determine how the patient's health condition has evolved in time (Obtain access to patient's clinical history and data goal), which positively contributes to establishment of the diagnosis.

Another common technique in Tropos is denominated contribution analysis. This technique highlights important social and/or ethical issues which are rarely captured by other kinds of analysis. For instance, Figure 3 documents a relevant issue that the physician takes into account when prescribing a drug. One of his/her softgoals refers to prescribing the most effective drug to treat a certain case 
(Prescribe the most effective drug goal). We have noticed that commonly the most effective drug is also the drug which causes multiple collateral effects (negative contribution of Prescribe the most effective goal towards Minimize collateral effects goal). Thus, when prescribing the treatment, the physician encounters these particular issues, requiring that he/she decides according to each case based on his/her self judgment and contact with the patient. When minimizing the patient's physical suffering (Minimize patient's physical suffering and symptoms goal) and the collateral effects, the physician positively contributes to the patient well-being (Guarantee patient well-being goal).

In the pursuit of the patient's health and well-being, there is also a need to integrate the department's service with other health care providers (Coordinate patient care with other healthcare providers goal). These involved providers are classified according to three types: other hospital departments, such as laboratories or administrative departments (Coordinate patient care with other hospital departments goal), municipal and state health services (Coordinate patient care with municipal and state health services goal) and specialists in areas related to rheumatology (Coordinate patient care with specialists in areas related to rheumatology goal). The integration of information between the rheumatology department and other departments which compose the public health care service is a fundamental quality factor for the success of the treatment, since the information must reach all decisionmakers who care about that information. From this point of view, being integrated with laboratories allows the physician to have updated information for selecting the proper treatment. Sharing information with municipal and state health services, on its turn, allows the physician to understand how the patient's health state is evolving along the time (to obtain what is called "integrated treatment"). And finally, coordinating the treatment with specialists in areas related to rheumatology allows the physician to clarify further details about the diagnosis with other specialists (for example, a dermatologist or ophthalmologist) in the hospital. To have integrated information helps to reduce uncertainty during the process of diagnosis (Eliminate all uncertainty during the process of diagnosis goal), helping the physician to deliberate about the treatment (although the access to information has a weak impact on reducing these uncertainties, as we explain latter).

Finally, with respect to the development of research achieved by the department, i.e. to investigate the incidence of rheumatologic conditions in population, the physician also has the Collect data for epidemiological analysis goal.

\subsection{Business process model: Rheumatologic Medical Consultation}

The process named Diagnose patient's health state has been modelled in the EPC diagram shown in Figure 4. In this diagram, the activities are arranged in swimlanes according to the actors who perform them. We omit events between sequential activities for space constraints. Also, to enable visualization of the whole process, we split the diagram in two and exhibit the two parts side-by-side in Figure 4.

This business process starts when the need for diagnosis is identified in another business process responsible for the selection of patients to be admitted by the department, i.e., after a patient is referred to the rheumatology department. The patient reports the current symptoms while the physician investigates the patient's clinical, personal and family history, and performs physical examination. These initial activities aim at diagnosing the patient's health state. According to what is reported by the patient, the physician decides whether laboratorial exams are required to formulate a diagnostic hypothesis or to confirm a diagnosis. In case exams are indeed required, the physician verifies whether they have already been requested in a previous consultation. If not, the patient is referred to a laboratory for examination. The patient is responsible for undergoing examination and forwarding the results of laboratorial exams to the physician. Eventually, the physician elaborates a diagnostic hypothesis. At this point, the physician identifies: (i) the existence of a serious rheumatologic condition; (ii) the existence of a mild rheumatologic condition (low complexity rheumatologic condition); or (iii) the absence of rheumatologic conditions. In the latter two cases, the physician releases the patient immediately. In the case of a serious rheumatologic condition, the physician explains the diagnostic hypothesis to the patient and decides whether the patient requires rheumatologic treatment. If not, then the physician releases the patient. In case a treatment is needed, he/she starts elaborating the therapeutic treatment, deciding about the most appropriate procedure to treat the patient and, finally, sending the patient to the proper clinic. We must highlight that due to capacity constraints, the department only admits patients with serious rheumatologic conditions. Patients with mild rheumatologic conditions are referred to the municipal health service for follow-up in basic health care units after 3 consultations.

\subsection{Results of the alignment in the context of the case study}

We have already discussed the main benefits of adopting the alignment between business process models and goal models. Since goal statements are not uniform, before being aligned with process models, the goal domain requires harmonization. The main aim of this section is to address the differences in the goal domain as well as to discuss how the different natures of goals impact on the structures of the business processes which support them. In the remainder of the section we propose a goal taxonomy to equalize the goal domain, the concepts involved in each of the categories of the taxonomy and finally, the implications in the business processes structures which support these goals. 


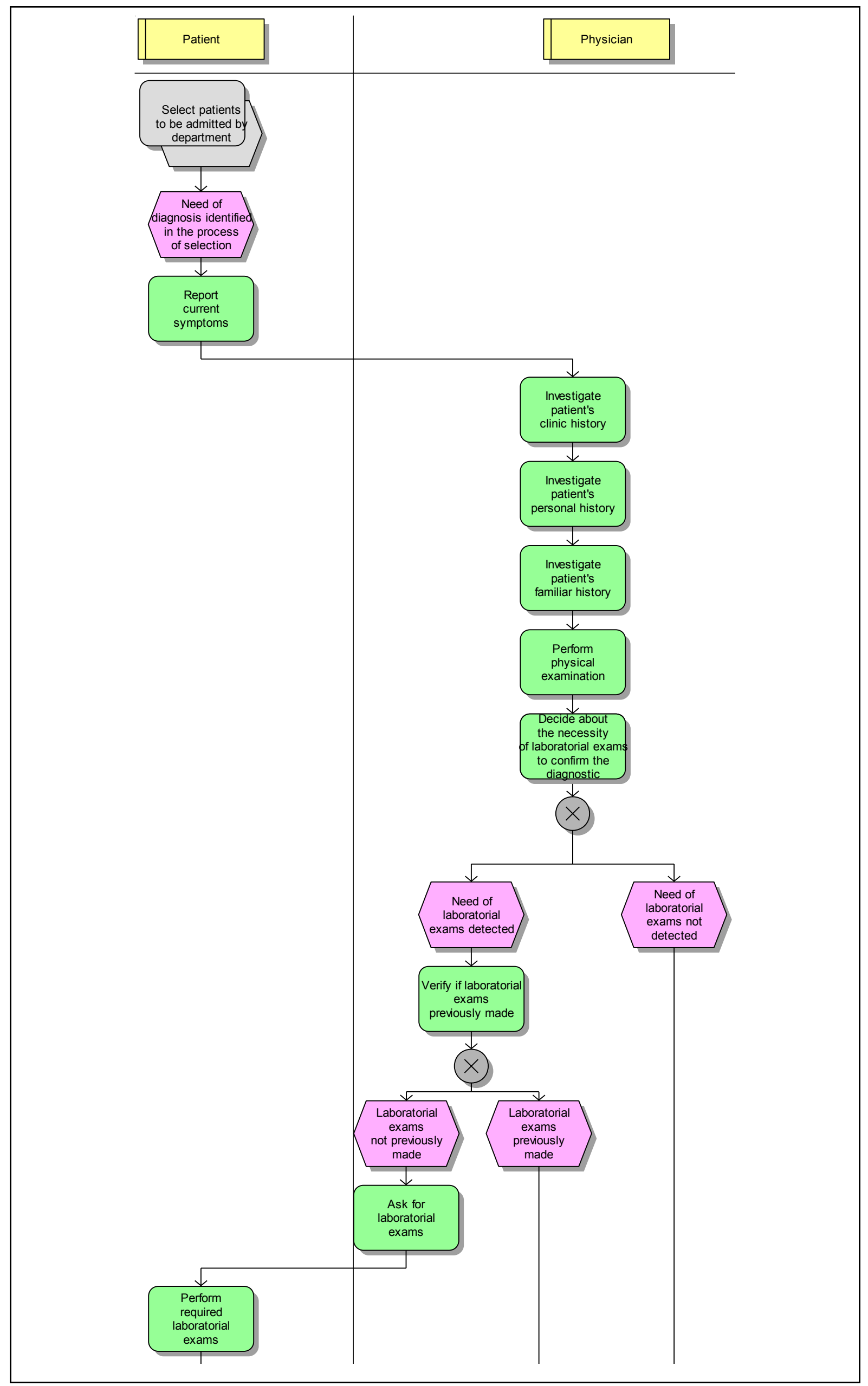




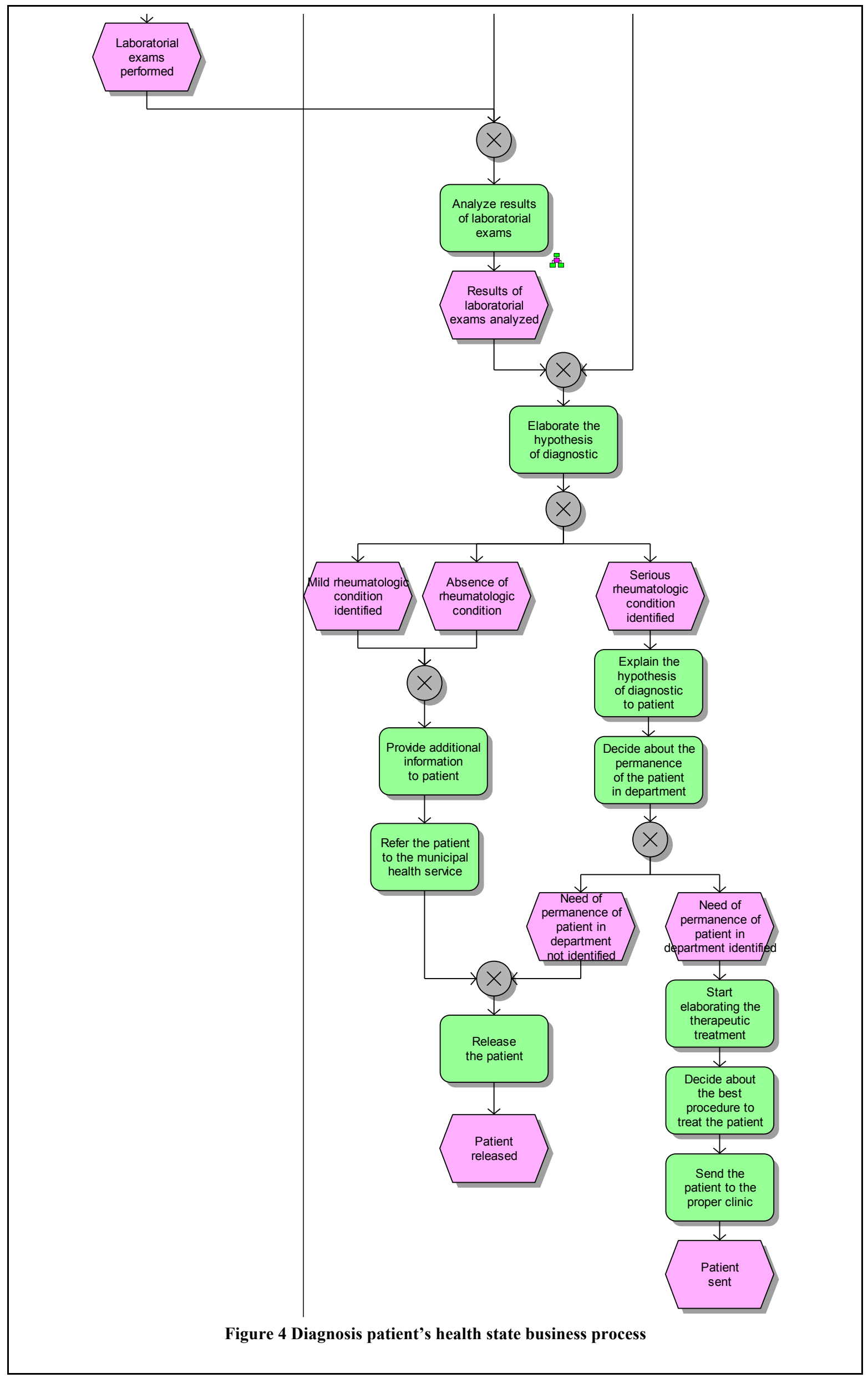




\subsubsection{Goal Taxonomy}

The first dimension we have noticed refers to the level of abstraction. Goals formulations range from highly abstract statements, such as "Provide the best treatment possible" to low level propositions such as "Medical reports must be issued within five days of frequency".

The classification proposed in [19][14] categorizes goals (or objectives, as denominated in the proposal) according to: a) fundamental objectives, which "describe business values" [18] and "concern the ends that decision makers value in a specific decision context" [14]; b) means-ends objectives, i.e. "means to achieve ends" [19] and "methods to achieve ends" [14]; and c) process objectives which are a subset of means objectives, i.e., "process objectives are ends towards achieving overall business objectives, as are the means objectives." [19]. Top-level means-ends objectives are refined until the lower-level means-ends objectives can be assigned to process objectives which implement them.

Here, we consider the same taxonomy (namely, fundamental goals ${ }^{1}$, means-ends goals and process goals), although our approach introduces some differences in relation to this classification.

First, this proposal assumes that means-ends goals do not have to be linked to individual fundamental goals as long as it is assumed that means-ends goals together are sufficient to achieve fundamental goals [19]. Instead, we regard that the relationships between means-ends goals and fundamental goals can be captured by the relations expressed in the Tropos metamodel (means-ends links, contribution links and AND/OR decomposition). Second, we introduce an additional kind of goal, namely, activity goals which we define as a desired state of the world which must be reached after the execution of the activity responsible for attaining this state.

As an example in the case study of the categories above mentioned, we have the following goals: Provide medical care to patient (fundamental goal), Provide medical care in scheduled medical consultation (means-ends goal), Diagnose patient's health state (process goal) and Prescribe patient's treatment (activity goal). We regard Provide medical care to patient as a fundamental goal since the main value considered by the organization during the decision making process is the provision of medical care. In this context, we regard fundamental goals as guidelines for driving the decision making process in the organization and, moreover, we introduce the notion that agents who are affiliated to the organization are always committed with a fundamental goal during the period of time which the agent belongs to this organization ${ }^{2}$. Provide

\footnotetext{
${ }^{1}$ Notice that the terms goal and objective are interchangeably used here.

${ }^{2}$ Observe that this definition opens up the discussion about the adoption of organizational goals by agents. In fact, we intend to address this issue in our future work. We aim at discussing the role of norms and rules as a regulator mechanism to enforce the agents' behaviour with the respect of which goals the agents adopt as well as how agents are constrained in the selection of the alternatives to attain these goals.
}

medical care through consultation is a means-end goal because consultation is a particular means to Provide medical care to patient. Since ends can be implemented by alternative means, in this case, an alternative solution to provide medical care is via emergency room (ER) (Provide medical care is via emergency room (ER) goal). Actually, although the ER is a feasible mean for providing medical care, physicians try to avoid this alternative at all costs, since providing medical care to patient via ER means that the patient's health state is so critic that the patient cannot wait until the next medical consultation, i.e. he/she must immediately receive medical care. Diagnose patient's health state is a process goal since there are multiple activities in the business process which contribute to achieving this goal (for example, the activities Investigate patient's clinical history, Investigate patient's personal history, Investigate patient's family history and Perform physical examination). It is also relevant to highlight that process goals sometimes can either be associated with a specific business process (for instance, the Diagnose patient's health state goal which is associated with the process of diagnosis) or can be partially satisfied within several business process simultaneously. For example, the Acquire technical skills goal is attained in various different business processes at the same time. While a diagnosis is obtained through several activities, prescribing treatment (Prescribe patient's treatment) is a goal of a specific activity in the business process (Start elaborating the therapeutic treatment activity). In the process of diagnosis, after the condition has been diagnosed, the physician starts elaborating the therapeutic treatment. At this point, the patient is forwarded to some specific clinic, where treatment will actually be elaborated and refined.

This dimension (functional/non-functional goals) has been identified in the software engineering domain in [11]. Functional goals refer to services that the organization environment is expected to deliver (i.e., what is achieved in fact), whereas non-functional goals refer to quality attributes that the organizational environment needs to satisfy while delivering the services (i.e., how the organization provides the services). In the case study, Diagnose rheumatologic conditions is a functional goal since it refers to what the business process is supposed to deliver (the patient's health state diagnosed), while Release patients with mild rheumatologic conditions after no more than 3 consultations is a non-functional goal since it refers to a quality attribute of the process (performance in terms of physician and patient resources which are allocated to a consultation).

The third dimension is also relevant in requirements engineering and is already supported by the Tropos metamodel. In this dimension, goals are categorized as either hardgoals or softgoals. Commonly, NFRs and softgoals are interchangeably treated as the same concepts (albeit the distinction is clear according to the definition of the concepts). This association arises because there is a tendency in specifying quality attributes in an imprecise manner. As examples of hardgoals/softgoals in the context 
of the case study, we have the Diagnose patient's health state goal and Select the most suitable treatment for patient goal, respectively. While Diagnose patient's health state is objectively defined in the context of the domain, Select the most suitable treatment for patient is not clear-cut defined a priori; it depends how the condition is responding to the prescribed treatment along the time as well as the acceptance of the treatment by the patient. The meaning of what is the most suitable treatment is specified in terms of the results obtained in the course of pursuing the goal.

The fourth dimension refers to what we call the scope aspect. In this dimension, goals are categorized according to the scope in which a goal may be fulfilled. A restrictedscope goal is a goal which should be achieved in a single execution of a business processes. A broad-scope goal is a goal which is attained after several executions of a business process. Notice that while fundamental and means-ends goals are always broad scope goals, activity goals are always restricted scope goals. Thus, this dimension is particularly relevant for process goals. Another important remark about this dimension is the fact that the multiple executions of the same business processes comprise in the service provided by the organization as perceived by the customer. To illustrate this distinction, consider the Approve the treatment proposed by the resident goal which is an example of a restricted scope goal. This goal is attained in multiple business processes in the organization, however, each process execution is independent; and for each patient, the physician has to validate the diagnosis issued by the resident. Collect data for epidemiological analysis is an example of broad scope goal. An analysis of the incidence of rheumatologic conditions in population needs sufficient data to allow a consistent decision-making in public policies for health care services. This amount of data is solely collected after a sufficient number of patients have been attended.

During the goal elicitation phase, the stakeholders commonly state strategies and motivations for the current business process. However, besides describing the current motivations, they also provide the motivations for altering the current organizational context as well as the motivations for a future organizational setting. Thus, the fifth dimension addresses temporal aspects. In the context of goal-oriented requirements engineering, [12] proposes four types of goals with respect to the temporal dimension: AS-IS goals, change goals, TO-BE goals and evaluation goals. We adopt the same classification, but we leave out evaluation goals (since these kind of goals are not relevant for our purposes). Then, we adapt these types of goals for the business process context. AS-IS goals concern the current organisational situation and how current organisational goals are realised in existing business processes (they provide motivations for the current business processes). TO-BE goals focus on statements that propose motivations and intentions in a future business context (the business processes which will exist in a future environment). Change goals refer to the reasons (the need) for altering the existing situation through the reengineering of current organizational setting. Although this kind of goal exists in a current business setting, driving the adoption of changes, they disappear as long as they are fulfilled.

As an example, consider the Approve the treatment proposed by the resident goal (classified as AS-IS goal). This goal is justified by a current organisational situation (the fact that resident's are still under training and are not legally responsible for treatment. The Coordinate patient care with other healthcare providers goal can be classified as a TO-BE goal ${ }^{3}$. This goal states that higher information integration among the involved stakeholders must be reached so that overall patient care becomes more efficient and effective. Finally, as a change goal, we have identified the Standardize diagnosis cue sheets goal which comprises an intention which is present in the current setting and the agents strives towards transforming the current situation. Thus, in a future situation, the cue sheets will be standardized and the need does not belong as a motivation for any action in a future setting, what lead to the disappearance of the goal. The difference between AS-IS goals and change goals is that the latter lead to alterations within the organization (what cannot be said about the former). The difference between TO-BE goals and change goals is that change goals are not present in the future setting (but in the current situation, instead) and disappear as they are attained.

Finally, the last differentiation we propose cannot be exactly classified as goals, but as desires [8]. A desire "refers to the 'will' of an agent towards a specific goal, although he/she might never actually pursue these goals". This has shown to be very relevant in addressing issues which exceed the boundaries of action of the actors within the organization. For instance, issues (goals) related with competitors or external partners cannot be controlled and thus, no action is available to achieve the goal. Another situation in which the concept of desire is valuable is related with the treatment of uncertainties in some organizational environment. In the case study, we have found that the Eliminate all uncertainty during the process of diagnosis is a desire of the physician. Rheumatology is an investigative speciality in which evidences must be considered in a whole clinic context. Thus, this "goal" denotes a desire of the physician to have the ability to cope with the subjectivity of evidences used to establish and validate a hypothesis of diagnostic. Although physicians would like to eliminate all uncertainty, there is no feasible plan to fulfil this goal.

We must also emphasize that classifying an objective either as a goal or as a desire is subjective. This classification must be driven by a number of issues, such as: the possibility of establishing commitments so that other agents pursue the goal, the possibility of associating concrete actions; or when there are no concrete actions, the

\footnotetext{
${ }^{3}$ Although this goal is a TO-BE goal, it is shown in Figure 3. In fact, we have decided to maintain the goal to illustrate its correlation with the Eliminate all uncertainty during the process of diagnosis goal.
} 
possibility of establishing strong correlations with plans and goals, among others. In this sense, although the goal Coordinate patient care with municipal and state health services to some extent impacts in the reduction of uncertainties during the process of diagnosis, this impact reveals to be so weak that we found relevant to classify the goal as a desire. Table 1 summarizes the proposed goal taxonomy.

\subsubsection{Implications of the Goal Taxonomy in Business Processes}

\section{Structures}

The level of abstraction of goals has a deep impact in the business processes structures that support them. In that respect, fundamental goals are not directly related with business process, instead, they are connected to means-ends goals and these, in turn, are connected with processes. The assignment of means-ends goals and process goals to process depends on the granularity of processes available in the organization. In other words, means-ends goals are decomposed in a finer goal structure until they can be assigned to process goals. This refinement is made up to the level of abstraction in which there exist processes to fulfil these (process) sub-goals. Finally, as denoted by the name, activity goals are connected with activities within the processes.

In the second dimension, while functional goals specify "what" must be achieved, leading to the adoption of actions (activities or business processes), commonly, non-functional goals guides the implementations of functional goals in organizational environments. Therefore, non-functional goals are not directly related with business processes; rather, they serve as guidelines or constraints during the implementation of functional goals, which in turn are associated with actions. As an example, we have the functional goal Prescribe patient's treatment which is associated with a specific activity and is characterized by the non-functional goal Select the most suitable treatment for patient. This latter constrains the treatment to be the best treatment possible, but is not associated with any specific activity.

Hardgoals or softgoals can both be associated with activities or business processes. In fact, the categorization of either hard or soft only refers to the specification of the goal (whether is possible to formulate the goal in sharply manner). This imprecision in the definition of the softgoal can not reveal a priori the structures which will support the goal, i.e., whether the goal will be attained by an activity, business process or a set of business processes. However, the solutions for attaining the softgoals are defined in the course of pursuing them [1615]. For example, with respect to the Acquire technical skills softgoal, the physician is not capable of defining how (s)he will acquire the knowledge a priori, this is defined as the physician provides attendance.

On one hand, since a restricted scope goal is directly related with one execution of some business process, by definition, this business process must be specific (and thus it is not possible to be attained by a set of business processes at the same time). On the other hand, a broad scope goal can be either achieved by one business process after it has executed for several times (e.g. Diagnose patient's health state goal) or by multiple business process after they have executed for several times (e.g. Acquire technical skills goal).

Temporal aspects also highly impact business process structures. They drive the creation, modification and

Table 1: Goal Taxonomy

\begin{tabular}{|c|c|c|}
\hline Dimension & Classification & Example \\
\hline \multirow{5}{*}{$\begin{array}{l}\text { Level of } \\
\text { Abstraction }\end{array}$} & Fundamental & Provide medical care to patient \\
\hline & Means-ends & Provide medical care in scheduled medical consultation \\
\hline & $\begin{array}{l}\text { Process (associated } \\
\text { with a specific } \\
\text { business process) }\end{array}$ & Diagnose patient's health state \\
\hline & $\begin{array}{lr}\text { Process } & \text { (partially } \\
\text { satisfied } & \text { within } \\
\text { multiple } & \text { business } \\
\text { process) } & \\
\end{array}$ & Acquire technical skills \\
\hline & Activity & Prescribe patient's treatment \\
\hline \multirow{2}{*}{$\begin{array}{l}\text { Functional/Non- } \\
\text { functional }\end{array}$} & Functional & Diagnose rheumatologic conditions \\
\hline & Non-functional & $\begin{array}{l}\text { Release patients with mild rheumatologic conditions after no more than } 3 \\
\text { consultations }\end{array}$ \\
\hline \multirow{2}{*}{$\begin{array}{l}\text { Hardgoals/ } \\
\text { Softgoals }\end{array}$} & Hardgoal & Diagnose patient's health state \\
\hline & Softgoal & Select the most suitable treatment for patient \\
\hline \multirow[t]{2}{*}{ Scope aspect } & $\begin{array}{l}\text { Restricted scope } \\
\text { goal }\end{array}$ & Approve the treatment proposed by the resident \\
\hline & Broad scope goal & Collect data for epidemiological analysis \\
\hline \multirow[t]{3}{*}{ Temporal aspect } & AS-IS goal & Approve the treatment proposed by the resident \\
\hline & Change goal & Standardize diagnosis cue sheets \\
\hline & TO-BE goal & Coordinate patient care with other healthcare providers \\
\hline Desire & & Eliminate all uncertainty during the process of diagnosis \\
\hline
\end{tabular}


extinction of organizational structures (in which business processes can be included). AS-IS goals present motivations for the existence of current organizational elements. Change goals formulate intentions which drive the organization towards reengineering. Thus, change goals can lead to the generation of new activities or business processes. Finally, TO-BE goals state about strategies in a future organizational context. Examples of these types of goals have been provided in the previous subsection.

At last, in the sixth dimension, the desires play an essential role in organizational modelling since they do not cause the creation of activities (or business processes) to be adopted by agents either to promote some positive impact in the achievement of some goal or to directly materialize the goal. In other words, desires do not establish relations with any element of the enterprise architecture. This absence of relations can be due to a broad number of reasons, such as: agents deem not relevant to pursue the goal when they have the opportunity, or the solution adopted to attain the goal is prohibitively costly or even due to the inexistence of actions to fulfil the goal.

\section{RELATED WORK}

Muscholl [1712] and Jablonski et al. [10] propose the integration of clinical pathways (CP) and Healthcare Information Systems (HIS), defining them as "an approach to connect knowledge about goals, tasks and medical and economic process". The main goal of a CP is to define a set of actions in the areas of diagnose, therapy, health care and administration to be realized in a determined medical treatment. Taking this model into consideration, medical and administrative tasks can be controlled as well as their costs and quality. Among the main advantages of integrating CPs, we can emphasize their use as a support for planning the calendar and resources of a medical treatment. In needing more complex systems, the planning can be fully automated. Besides, the documentation of an applied CP serves as support for posterior analysis about the success or failure of a determined treatment and to compare the outcomes of applying the same treatment in different institutions. This proposal resembles the work here presented in the sense that both adopt a process-oriented view to treat the medical domain. However, our focus differs from this work since, while we adopt a detailed representation of business processes (in which we capture the information systems which support the business processes, but also strives to address business aspects independently from specific systems), this approach solely addresses aspects related with information systems which support business processes.

Perini et al. [20] describe a case study in a health care environment using the Tropos methodology to conduct what the authors call intentional analysis. We notice that this kind of analysis is strongly grounded in goal analysis, focusing on the intentional elements available in Tropos, such as: the dependencies among actors and the plans and resources utilized by them. In their study, they have analyzed different alternatives in order to fulfil actors' goals (in this case a group of nurses). This analysis enables one to restructure the organization in order to better fulfil actors' goals. However, the authors do not cope with a detailed representation of business processes involved in the case study, restricting their solution to a proposal of a goal model expressed in the Tropos language.

Concerning the integration between business processes and goals, Neiger and Leonid [19] propose an approach for goal-oriented business process analysis. They integrate the business process domain (in which business process are also modelled in EPC diagrams) and the goal domain which is represented, in the decisions sciences scope, by what is denominated as "Value Focused Thinking" (VFT) framework. The goal of this initiative is to establish a relation between goals (captured in the VFT framework) and analysis methods to facilitate the decision making process to be applied to business processes, so that efficiency and effectiveness issues are properly addressed. An interesting aspect of that work is that it is used of formalization for goal analysis. Although there are initiatives in formal reasoning in the Tropos methodology, we have not yet applied these techniques. With respect to the goal taxonomy, Neiger and Leonid have addressed a single dimension (level of abstraction) concerning to the goal nature, distinguishing between fundamental goals, means-end goals and process goals. We have further refined the level of abstraction with an additional level (activities goals) and, moreover, we have extended the goal taxonomy by proposing other five dimensions for goals.

Andersson et al. [1] have also addressed the alignment between business processes and goals. Their method receives as input an AS-IS business model formulated in terms of the REA Ontology (Resource-Event-Agent) and a TO-BE goal model (formulated in the $i^{*}$ language with the support of the BMM - Business Motivation Metamodel) and delivers a TO-BE business process model which conforms to the TO-BE goal model. The method grounds in the notion of "mean" to establish a link between goal models and business models. The alignment between the AS-IS business models and the TO-BE goal models is made through "means templates" and "transformation rules" in order to produce TO-BE business models. In this approach, models are iteratively aligned during the modelling phase. Hence, after the modelling stage, business models and goal models are aligned. In the same way, to deliver TO-BE business models is necessary that both models, TO-BE business models and TO-BE goal models are aligned during the application of the methodology. This approach differs from ours because we construct goal models and business process models separately; the alignment between them is achieved in a subsequent stage.

\section{CONCLUSIONS}

This paper discusses the alignment of goal analysis methods (using the Tropos methodology) and business process modelling methods (using the ARIS framework). We propose a goal taxonomy to harmonize the goal domain to 
be subsequently aligned with business processes. Furthermore, we discuss the implications of this classification in establishing the relationships of the goal domain with the business processes domain. This alignment with goals models extends traditional business process methodologies by providing a dimension of intentionality to the business processes [13]. This contrasts with several approaches in business process modelling which focus on "how" business processes are performed (adopting a behavioural description in which business processes are solely described in terms of their procedural aspects).

We have observed that aligning process models and goal models is not straightforward. In the course of the case study, we have identified the need to split this effort into three phases: the elicitation phase (in which goal models and business process models are captured through interviews and observation), the harmonization phase (in which the goal domain is structured for alignment according to the business processes structures that will support it) and the alignment phase (in which the relationships between the goal domain and the elements of the organizational domain are established and in which the semantics of these relationships are elaborated). The first phase has already been conducted and the results about the goal elicitation sub-phase have been reported in [6]. The harmonization phase is addressed in this paper.

We have demonstrated the need for harmonizing the goal domain since different natures of goals impacts in the business processes structures which support these goals. In this sense, we have proposed six dimensions to classify goals (referring to level of abstraction, functional/nonfunctional aspect, hardgoal/softgoal, scope aspect, temporal aspect and desires). Moreover, we have provided a discussion about how each of these dimensions impact on the (re)design of business processes. Our main observation regarding goal harmonization is that different natures entails in different business process structures which support the goal domain. As some examples of these structures, we have shown that some kinds of goals do not lead to the generation of business process to support them (such as fundamental goals and desires). Other types of goals cope with a number of issues, such as the scope in which it must be fulfilled (if it sums up to a specific execution of the business process or the goal attainment is associated with the service provided by the organization as a whole), whether the goal refers to a current or future organizational situation, among others.

In our future work, we envision the extension of the Tropos meta-model to accommodate the proposed goal taxonomy. Besides, we intend to investigate suitable modelling notation and semantics to relate goal models and business process models. We also believe that Tropos models can be used to support formal reasoning during the selection of which strategy will be adopted to attain a specific goal. This should be fruitful in the systematization of business process reengineering.

With respect to computational tools, business process models allow the identification of which business process can be supported by information systems. In particular, two lines of research have shown to be promising: (i) the development (or acquisition) of workflow management systems (from the business process models) which manage the flow of documents in the department and (ii) the development (or acquisition) of knowledge management systems to promote the use and sharing of knowledge in health care institutions.

\section{ACKNOWLEDGEMENTS}

This work was partially supported by FAPES in the scope of the INFRA-MODELA project and in the scope of grant DCR number $37274554 / 2007$. We thank all physicians, interns, residents and patients at the Cassiano de Moraes University Hospital for their cooperation in this research. In particular, we are grateful to Prof. Dr. Valéria Valim and Érica Serrano, MD, for providing invaluable assistance in the execution of this research. We also thank Giancarlo Guizzardi for fruitful discussions in the scope of this research.

\section{REFERENCES}

1 Andersson, B., Bergholtz, M., Edirisuriya, A., Ilayperuma, I., Johannesson, P. and Zdravkovic, J. 2007 On the Alignment of Goals Models and Business Models. In REA-25. A Celebration of the REA Enterprise Model, Geerts, G., University of Delaware. Department of Accounting \& MIS.

2 Bresciani, P., Perini, A., Giorgini, P., Giunchiglia, F., Mylopoulos, J. 2004 Tropos: An Agent-Oriented Software Development Methodology. Autonomous Agents and MultiAgent Systems.

3 Bresciani, P. and Sannicolo, F. 2002 Requirements Analysis in Tropos: a self referencing example. Technical Report DIT02-105, University of Trento.

4 Cardoso, E., Almeida, J. and Guizzardi, G. 2008 Requirements Engineering Based on Business Process Models: A Case Study. XI Iberoamerican Workshop on Software Environments and Requirements Engineering (in Portuguese).

5 Cardoso, E. S., Guizzardi, R. S. S. 2008 Aligning Goal Analysis and Business Process Modelling: A Case Study in the Health Care Environment. Proceedings of II Workshop of Business Process Modelling in conjunction with XIV Brazilian Symposium of Multimedia Systems and Web, v.2, p.215-224, Vila Velha/ES, Brazil (in Portuguese).

6 Cardoso, E., Almeida, J.P.A., Guizzardi, G., Guizzardi, R.S.S. 2009 Eliciting Goals for Business Process Models with NonFunctional Requirements Catalogues, 10th International Workshop on Business Process Modelling, Development and Support (BPMDS 2009), Amsterdam, The Netherlands. Lecture Notes in Business Information Processing, SpringerVerlag.

7 Giorgini, P., Mylopoulos, J., Nicchiarelli, E. Sebastiani, R. 2004 Formal Reasoning Techniques for Goal Models. In Journal of Data Semantics.

8 Guizzardi, R.S.S., Guizzardi, G., Perini, A., Mylopoulos, J, 2007 Towards an Ontological Account of Agent Oriented Goals, Software Engineering for Multi-Agent Systems, Vol. V, Springer-Verlag, Berlin.

9 Halleux, P., Mathieu, L., Andersson, B. 2008 A Method to Support the Alignment of Business Models and Goal Models. 
In Proceedings of 3rd Intl. Workshop on Business/ITAlignment and Interoperability (BUSITAL'08).

10 Jablonski, S., Meiler, C., Müller, S., Lay, R. Process Oriented Knowledge Management to Support Clinical Pathway Execution 2005 In Proceedings of the Workshop on Current Aspects of Knowledge Management in Medicine (KMM05), Kaiserslautern, Germany.

11 Jureta, I. J., Faulkner, S., Schobbens P. Y. 2006 A More Expressive Softgoal Conceptualization for Quality Requirements Analysis. In Conceptual Modelling - ER, 25th International Conference on Conceptual Modelling, Arizona.

12 Kavakli, E. 2002 Goal-Oriented Requirements Engineering: A Unifying Framework." Requirements Engineering Vol. 6 No. 4, pp. 237-251.

13 Kavakli, E., Loucopoulos, P. 2003 Goal Driven Requirements Engineering: Evaluation of Current Methods. In Proceedings of the 8th CAiSE/IFIP8.1 Workshop on Evaluation of Modelling Methods in Systems Analysis and Design, EMMSAD.

14 Keeney, R. 1994 Creativity in Decision Making with ValueFocused Thinking. Sloan Management Review Summer pp. 33-41.

15 Kueng, P., Kawalek, P. 1997 Goal-based business process models: creation and evaluation. In Business Process Management Journal 3 pp. 17-38.

16 Lamsweerde, A. 2001 Goal-Oriented Requirements Engineering: A Guided Tour, 5th International Symposium on Requirements Engineering, IEEE Computer Society Press.

17 Muscholl, M. 2005 The "Integrated Clinical Pathways"Approach - Current Requirements to the Knowledge Management in Health Information Systems. In Proceedings of the Workshop on Current Aspects of Knowledge Management in Medicine (KMM05), Kaiserslautern, Germany.

18 Mylopoulos, J., Chung, L., Yu, E., Nixon, B. 1992 Representing and Using Non-functional Requirements: A Process-Oriented Approach, IEEE Trans. on Software Engineering, 18(6), pp. 483- 497.

19 Neiger, D., Leonid, C. 2004 Goal-Oriented Business Process Modelling with EPCs and Value-Focused Thinking. Business Process Management, pp. 98-115.

20 Perini, A., Bresciani, P., Yu, E., and Molani, A. 2004 Intentional Analysis for Distributed Knowledge Management. In van Elst, L., Dignum, V., and Abecker, A., editors, AgentMediated Knowledge Management, volume 2926 of LNAI, pages 351-367. Springer-Verlag, Heidelberg, Germany.

21 Scheer, A.W. 2000 Aris - Business Process Modelling, Springer-Verlag, New York.

$22 \mathrm{Yu}$, E. 1995 Modelling Strategic Relationships for Process Reengineering. PhD. Thesis, Department of Computer Sciences, University of Toronto. 